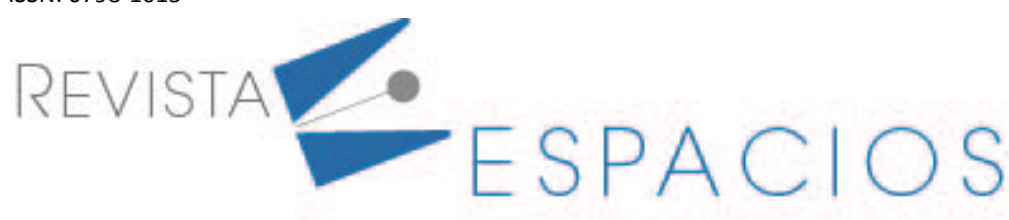

Vol. 42 (10) $2021 \cdot$ Art. 7

Recibido/Received: 14/02/2021 • Aprobado/Approved: 25/04/2021 • Publicado/Published: 30/05/2021

\title{
Estudio multidimensional de la pobreza en las familias del sector de Monte Sinaí-Guayaquil
}

\section{Multidimensional study of poverty in families in the Monte Sinaí-Guayaquil}

\author{
MAZAIRA, Zahily ${ }^{1}$ \\ ALONSO, Irán² \\ VARGAS, Andrea V. ${ }^{3}$ \\ BARRETO ZÚÑIGA, William W. ${ }^{4}$
}

\begin{abstract}
Resumen
El presente artículo sintetiza un estudio multidimensional de la pobreza en las familias del sector de Monte Sinaí-Guayaquil, Ecuador. Implementando parámetros para llegar a medir la percepción de las familias con respecto a la pobreza y calidad de vida. La investigación tiene un enfoque cuantitativo, utilizando métodos de análisis - síntesis, histórico - lógico e inductivo y deductivo; presentados estos en el proceso para el cálculo del índice. Obteniendo indicadores que demuestran el tipo de pobreza que mantienen las familias del sector.
\end{abstract}

Palabras clave: pobreza, familias, indicadores.

\begin{abstract}
The present work carries out a multidimensional study of poverty in families in the Monte SinaiGuayaquil, Ecuador, implementing parameters to measure the perception of families regarding poverty and quality of life. The research has a quantitative approach, using methods of analysis - synthesis, historical - logical and inductive and deductive, presented in the process for calculating the index. Obtaining indicators that demonstrate the type of poverty that families in the sector maintain.
\end{abstract}

key words: poverty, families, indicators.

\section{Introducción}

Con el pasar de los años y la evolucion de la sociedad, la pobreza ha recibido diferentes conceptualizaciones; $\sin$ embargo, para el presente estudio, se la define como un estado de indigencia general, en el cual las personas no disponen de recursos para satisfacer sus necesidades básicas, dos factores que favorecen el crecimiento de la pobreza son la falta de equidad y de igualdad. La pobreza supone la degradación del ser humano y constituye una afectación directa al derecho del Buen Vivir, concepto que se popularizó en el Ecuador durante el mandato del Econ. Rafael Correa, la esencia del Buen Vivir es la búsqueda constante del desarrollo sustentable y sostenido,

\footnotetext{
${ }^{1}$ Doctor en Ciencias Económicas, Master en Administración de Negocios, Profesora Titular Agregado 2 de la Facultad de Ciencias Económicas, Universidad de Guayaquil. email: zahily.mazairar@ug.edu.ec

${ }^{2}$ Master en Desarrollo Socio Económico Local, profesor de la Facultad de Ciencias Económicas, Universidad de Guayaquil. email: iran.alonsoh@ug.edu.ec

${ }^{3}$ Economista. email: a_vargas14@hotmail.es

${ }^{4}$ Economista. Formador de Formadores. Email: williambz@hotmail.es
} 
a través del cual se posibilite la erradicación de la pobreza, y así poder brindar una mejora en la calidad de vida de los habitantes.

Con la finalidad de que se dé prioridad a los sectores más vulnerables de la sociedad, se emiten los Objetivos del Milenio, partiendo del hecho de que es obligación de toda nación que se acoja a los preceptos del sumak kawsay o buen vivir, mantener los niveles de equidad en la vida de los ciudadanos. Con base en lo antes mencionado en el año 2013 se formula el Plan Nacional del Buen Vivir, donde se adaptan los Objetivos del Milenio, con la finalidad de brindar mayor profundidad a las políticas sociales, hasta el punto de convertirlas en una realidad.

“El buen vivir es la forma de vida que permite la felicidad y la permanecía de la diversidad cultural y ambiental es armonía, igualdad, equidad, y solidaridad. No es buscar la opulencia ni el crecimiento económico infinito"(Secretaria Nacional de Planificación y Desarrollo - Senplades, 2013). En este plan se describen de manera estructurada las metas relacionadas con la erradicación de la pobreza y sus respectivos lineamientos, con lo cual, la pobreza se convierte en uno de los principales indicadores para determinar la calidad de vida de las personas.

Con base en lo antes mencionado, se puede acotar que existen sectores específicos en los cuales sus habitantes viven en condiciones de pobreza e indigencia. Para el presente estudio se decide analizar la problemática de Monte Sinaí que es una comunidad ubicada en el norte-este de Guayaquil, donde existe un alto porcentaje de familias que viven en condiciones de pobreza y extrema pobreza, según el censo de condiciones de vida realizado en el año 2014; este es uno de los sectores más vulnerables de la ciudad, presentando características que le impiden gozar de un buen vivir.

Entre las causas que dieron origen a este problema, están los bajos niveles de educación de la población y la mala calidad de vida, que desembocan en una pérdida de competitividad en el mercado laboral, lo que conlleva que estas personas tengan menos oportunidades de acceder a una plaza de empleo digno. La explotación de la población conduce a que el capital se acumule en los sectores más privilegiados, dando origen a las desigualdades, haciendo que las personas no gocen de beneficios y se les imputen privaciones, con lo cual se les niega una oportunidad para alcanzar condiciones de desarrollo personal. Por último, está el deterioro de la calidad de vida de las personas, con lo cual se da paso a la delincuencia, prostitución, drogadicción, alcoholismo y la violencia familiar.

Se considera necesario realizar un estudio al sector de Monte Sinaí basado en un Índice de Pobreza Multidimensional (IPM), con lo cual se posibilitaría la determinación de los factores que alientan al crecimiento de los casos de familias en condiciones de pobreza y extrema pobreza para, con base en los resultados, formular soluciones efectivas. Finalmente, se tiene que este estudio, sería de gran utilidad para las entidades públicas competentes, puesto que, podrán apreciar dicha realidad y priorizar la implementación de nuevas políticas para que estas familias tengan una buena calidad de vida, de manera que puedan desarrollarse como ciudadanos, generando progreso para el sector.

Con base en lo antes mencionado se establece que el objetivo general de la investigación es determinar un índice multidimensional de pobreza para las familias del sector Monte Sinaí, que permita la identificación de las causas socioeconómicas de su situación. A continuación se presentan los objetivos específicos, mismos que son necesarios para cumplir las generalidades del proyecto:

- Sistematizar los conceptos, teorías y métodos de medición de la pobreza.

- Establecer el procedimiento metodológico para la determinación del índice multidimensional de pobreza

- Medir la situación de pobreza en el sector Monte Sinaí, identificando sus causas socioeconómicas, utilizando para ello el índice propuesto en la investigación. 
- Comparar los resultados del índice de pobreza multidimensional (IPM) con el índice de pobreza común en la determinación de la pobreza social.

\subsection{La pobreza, una visión desde las principales escuelas de pensamiento económico}

Contando con una perspectiva histórica es posible abordar la pobreza desde el punto de vista de diferentes escuelas del pensamiento económico, por lo que a continuación se presentan las diferentes maneras en que estas solventaron la pobreza:

La pauperización fue de la mano con el desarrollo capitalista, desde el siglo XIV en la Europa preindustrial, con el tránsito del feudalismo a la Edad moderna se generó una revaloración de los pobres como un problema social, se comenzaron a dictar medidas para remediar el mal social, agravado como efecto de la Peste Negra.

El mercantilismo fue el manejo económico que imperó durante la Edad Moderna, con el desarrollo del Estado absolutista fundado en la centralización, el poder soberano, las burocracias administrativas, fiscalidad, instituciones que garantizaron un control sobre la producción y los precios.

La escuela de pensamiento clásico también destaca en los estudios de pobreza, citando las aportaciones de Adam Smith, Tomas Malthus y Karl Marx. A continuación, se expondrán algunas de sus ideas sobre el tema objeto de esta investigación. En lo que respecta a Adam Smith, este definía a los pobres como personas que apenas podían satisfacer sus necesidades básicas de subsistencia, esto como consecuencia de la desigualdad. Este veía la pobreza como un proceso histórico y tomando en consideración que la mayor parte de la población estaba constituida por criados, trabajadores y operarios, expresó que ninguna sociedad es floreciente cuando la mayor parte de la población son pobres o miserables.

En el caso de Malthus, promovió los cambios de la Ley de los pobres y la Ley de Asentamientos en Inglaterra, entre los mecanismos contenidos en estas leyes están los subsidios, los cuales en su criterio no harían otra cosa sino aumentar los precios, conforme aumente la demanda de ingresos adicionales se causaran afectaciones en el aumento de la producción.

Para Marx analiza la pobreza desde una perspectiva política, donde acusa al sistema económico de tratar a la fuerza de trabajo como una mercancía más, esto debido a que durante el crecimiento industrial, la demanda de mano de obra era considerable y era común ver campesinos desempleados y desposeídos, en dicha tendencia se apreció la apropiación de los medios de producción, donde se incluyen la concentración de la riqueza, la división del trabajo y el desarrollo de las fuerzas productivas.

\subsection{Medición de la pobreza en el Ecuador}

En el Ecuador la medición de la pobreza se realiza a través de tres métodos, el del ingreso, el de Necesidades Básicas Insatisfechas o por medición multidimensional. Esta labor es realizada por el Instituto Nacional de Estadística y Censo (INEC), de manera anual y semestral; para proceder con los cálculos de estos índices es necesario contar con las cifras estadísticas de la Encuesta Nacional de Empleo, Desempleo y Subempleo (ENEMDU). A continuación se presentan los resultados del comportamiento de la pobreza en el Ecuador según los métodos antes mencionados:

Actualmente el índice de pobreza y desigualdad por ingreso, se obtiene con la actualización de la línea oficial de pobreza por consumo mediante el Índice de Precios al Consumidor (IPC); quienes posean un ingreso total per cápita por debajo de la línea de pobreza reciben la consideración de pobres, con lo cual se calcula la proporción de pobres frente a la población total (Serrano, 2017). 
En diciembre de 2017 la línea de pobreza se ubicó en US\$ 84,49 mensuales por persona, mientras que la línea de pobreza extrema en US\$ 47,62 mensuales per cápita, como se muestra en Gráfico 1. Con este umbral, a nivel nacional la incidencia de la pobreza es del $21,5 \%$ y la extrema pobreza del $7,9 \%$. A nivel urbano la incidencia de la pobreza es del $13,2 \%$ y la extrema pobreza del 3,3\%, mientras que en el área rural la pobreza es del 39,3\% y la pobreza extrema del $17,9 \%$.

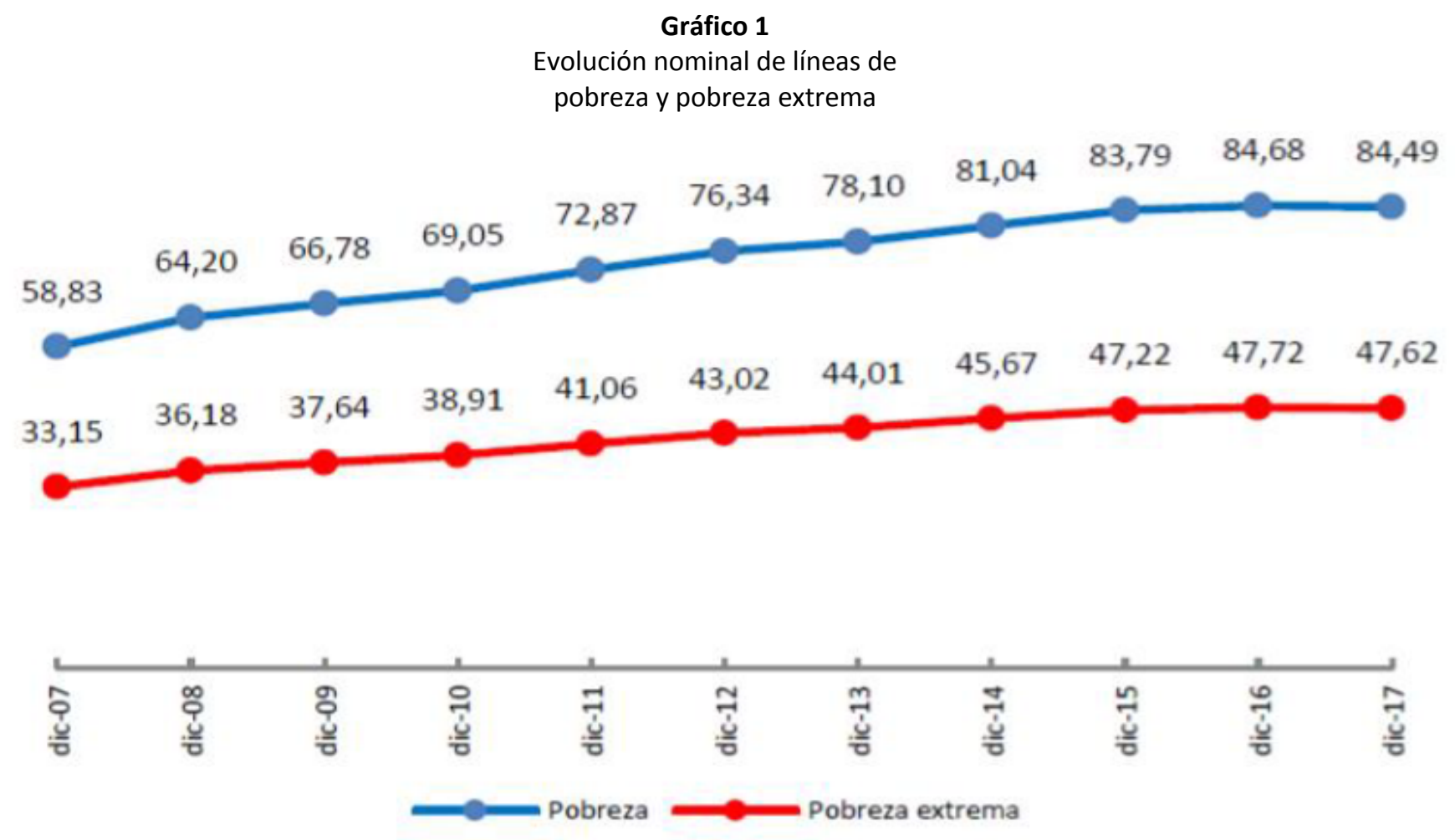

Fuente: Informe de pobreza y desigualdad - 2017

En cuanto al método de medición de la pobreza por Necesidades Básicas Insatisfechas (NBI), se puede acotar que es un modelo introducido por la CEPAL en 1981, mismo que fue adaptado a la realidad del Ecuador y se ejecuta con base en cinco componentes, entre los cuales se cuentan la calidad de la vivienda, el hacinamiento, acceso a servicios básicos, educación y capacidad económica.

Si el hogar carece de al menos uno de los componentes sus miembros son considerados pobres por NBI. En el Gráfico 2, es posible apreciar que a nivel nacional la tasa de pobreza por NBI en diciembre 2017 se ubicó en $31,8 \%$. En un análisis más específico es posible evidenciar que en el área urbana la pobreza por NBI es de 20,5\% mientras que en el área rural fue de 56,1\%. En relación al mismo mes en 2016 no se evidencia una diferencia estadísticamente significativa. De los componentes de las NBI, la falta de acceso a servicios básicos de agua y eliminación de excretas, son las que presentan tasas más altas, hasta diciembre de 2017 , esta fue de $18,2 \%$ a nivel nacional, en cuanto al área rural en 2017 se registró una tasa de 45,1\%, considerablemente alta en comparación con la del área urbana que fue de 5,7\%. 


\section{Gráfico 2}

Evolución de la tasa de pobreza por necesidades básicas insatisfechas (NBI)

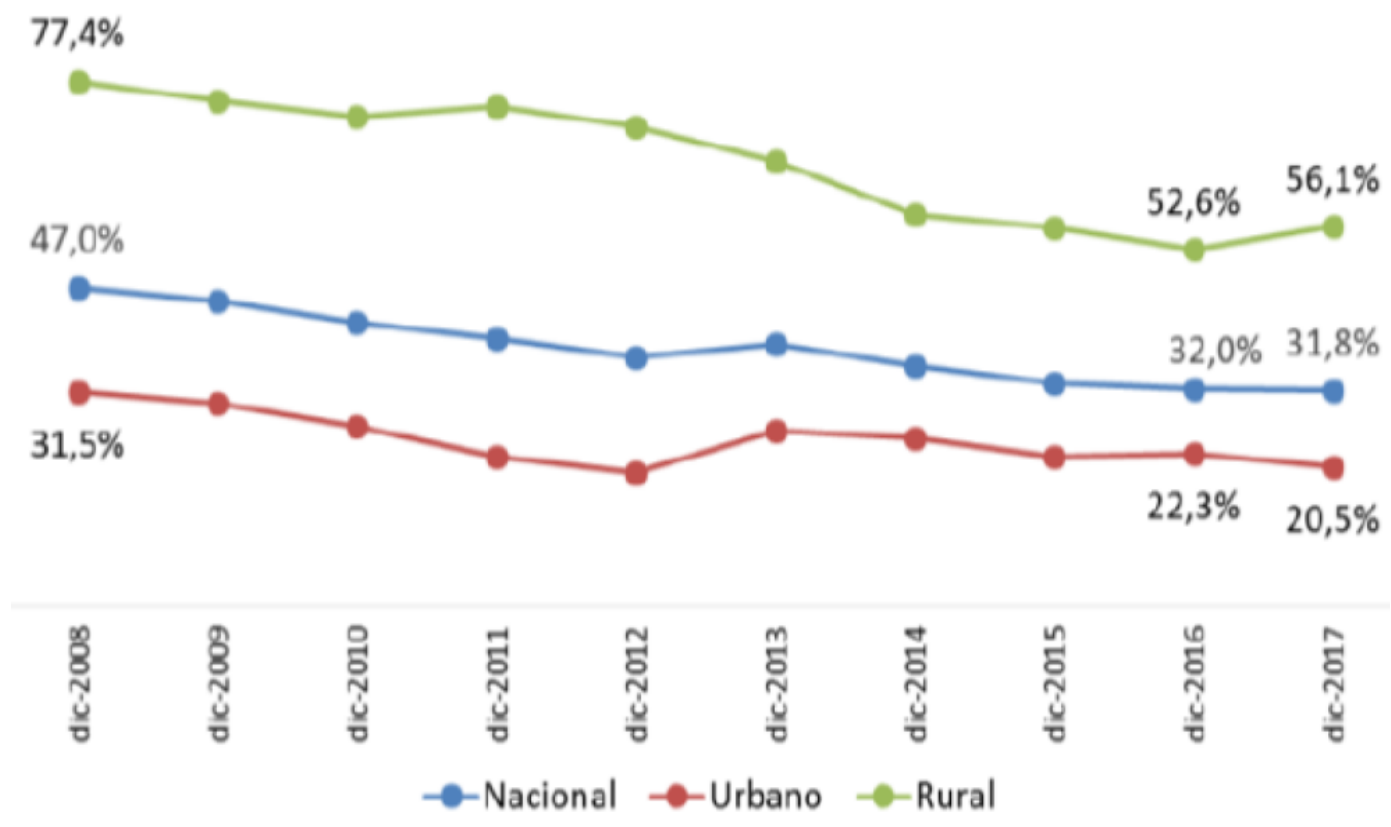

Fuente: Informe de pobreza y desigualdad - 2017

Por último está la medición de la pobreza multidimensional, Ecuador para dicha medición recurre al Método desarrollado por Alkire y Foster en el 2007 que permite evaluar de manera simultánea las diferentes privaciones o vulneraciones de derechos a los cuales se enfrentan los hogares ecuatorianos. Este método aborda el problema de identificación y agregación (SEN, 1976).

Para identificar a las personas pobres multidimensionales se aplica un criterio de corte dual, como el que se presenta en la Cuadro 1, donde es posible identificar dos etapas. En la primera, se construye un set de indicadores donde se fija un umbral de privación por cada indicador. En la segunda etapa, se define un punto de corte que determina el número de privaciones que requiere una persona para ser considerada como pobre y pobre extrema.

La construcción de las siete dimensiones presentadas en el Cuadro 1, parte de la interpretación estadística del Capítulo segundo del Buen Vivir de la Constitución (Artículos 12 al 34); esto permite establecer la privación del derecho en cada ámbito y asociarlo con la información estadística existente para alcanzar una convergencia entre la privación conceptual y el indicador construido (Añazco \& Pérez, 2016).

Bajo estas premisas y considerando como ya se indicó con anterioridad la metodología de la medición del IPM se toma de referencia la encuesta de empleo y subempleo; ENEMDU elaboradas por el INEC, la misma que tiene una cobertura urbana y rural cada año.

Esta encuesta posee una serie de módulos que permiten analizar y monitorear la evolución del mercado laboral ecuatoriano, así como ciertas características de las condiciones de vida de la población, por ejemplo, vivienda, ingresos, educación y seguridad social. La encuesta de empleo es la fuente de información más periódica que existe en el Ecuador y de ella se obtienen las cifras oficiales de pobreza por ingresos, mercado laboral y educación. (Dávila, S. B.; Ortega, F. C., 2015). 


\section{Cuadro 1}

Dimensiones e Indicadores del Índice de

Pobreza Multidimensional- IPM- del Ecuador

\begin{tabular}{|c|c|c|c|c|}
\hline Dimensión & Pesos & & Indicador & $\begin{array}{l}\text { Población } \\
\text { aplicable }\end{array}$ \\
\hline \multirow{3}{*}{$\begin{array}{l}\text { Educación } \\
\qquad(25 \%)\end{array}$} & $8.3 \%$ & 1. & $\begin{array}{l}\text { Inasistencia a } \\
\text { educación básica y } \\
\text { bachillerato }\end{array}$ & S a 17 años \\
\hline & $8.3 \%$ & 2. & $\begin{array}{l}\text { No acceso a } \\
\text { educación superior } \\
\text { por razones } \\
\text { económicas }\end{array}$ & 18 a 29 años \\
\hline & $8.3 \%$ & 3. & $\begin{array}{l}\text { Logro educativo } \\
\text { incompleto }\end{array}$ & 18 a 64 años \\
\hline \multirow{3}{*}{$\begin{array}{l}\text { Trabajo y } \\
\text { Seguridad } \\
\text { social }(25 \%)\end{array}$} & $8.3 \%$ & 4. & $\begin{array}{l}\text { Empleo infantil y } \\
\text { adolescente }\end{array}$ & 5 a 17 años \\
\hline & $8.3 \%$ & 5. & $\begin{array}{l}\text { Desempleo o empleo } \\
\text { inadecuado }\end{array}$ & $\begin{array}{l}18 \text { años y } \\
\text { más }\end{array}$ \\
\hline & $8.3 \%$ & 6. & $\begin{array}{l}\text { No contribución al } \\
\text { sistema de pensiones }\end{array}$ & $\begin{array}{l}15 \text { años } \mathrm{y} \\
\text { más }\end{array}$ \\
\hline \multirow{2}{*}{$\begin{array}{l}\text { Salud, Agua y } \\
\text { Alimentación } \\
\qquad(25 \%)\end{array}$} & $12.5 \%$ & 7. & $\begin{array}{l}\text { Pobreza extrema por } \\
\text { ingresos }\end{array}$ & $\begin{array}{l}\text { Toda } \\
\text { población }\end{array}$ \\
\hline & $12.5 \%$ & 8. & $\begin{array}{l}\text { Sin servicio agua por } \\
\text { red pública }\end{array}$ & $\begin{array}{l}\text { Toda } \\
\text { población }\end{array}$ \\
\hline \multirow{4}{*}{$\begin{array}{l}\text { Hábitat, } \\
\text { Vivienda y } \\
\text { Ambiente } \\
\text { sano }(25 \%)\end{array}$} & $6.25 \%$ & 9. & Hacinamiento & $\begin{array}{l}\text { Toda } \\
\text { población }\end{array}$ \\
\hline & $6.25 \%$ & 10. & Déficit habitacional & $\begin{array}{l}\text { Toda } \\
\text { población }\end{array}$ \\
\hline & $6.25 \%$ & 11. & $\begin{array}{l}\text { Sin saneamiento de } \\
\text { excretas }\end{array}$ & $\begin{array}{l}\text { Toda } \\
\text { población }\end{array}$ \\
\hline & $6.25 \%$ & 12. & $\begin{array}{l}\text { Sin servicio de } \\
\text { recolección de basura }\end{array}$ & $\begin{array}{l}\text { Toda } \\
\text { población }\end{array}$ \\
\hline
\end{tabular}

Fuente: Informe de pobreza y desigualdad- 2017

Ahora bien considerando la propuesta del IPM en el Ecuador, cabe recalcar ciertos parámetros por los cuales este índice delimita lo que está midiendo, como por ejemplo; este índice solo plantea 4 dimensiones, con 12 indicadores los cuales en su mayoría, siguen midiendo la pobreza monetaria mas no, otro tipo de pobreza que pueden llegar a tener las familias, no tiene indicadores que midan perspectiva de pobreza en cada familia, porque se sabe que eres pobre, pero cada familia que tan pobre se considera.

(Cruz, 2016), realiza una crítica a dicho índice donde plantea que, la conceptualización de la pobreza no puede ser reducida a un número (o conjunto de números) sin que estos no representen un concepto consensuado de pobreza. El IPM propone 4 dimensiones con 12 indicadores, reducidos a un solo índice que cuando sea leído por el hacedor de política podría asumir que sus componentes -en conjunto- reportan un avance o retroceso. Eso es un error peligroso. El proceso de construcción de una estadística es más importante que la estadística misma. ¿Quién decidió los 12 subindicadores incluidos? ¿Por qué no 13 ? ¿Por qué se les otorgó un peso de $6 \%$ a unos y $12 \%$ a otros? ¿Por qué el déficit habitacional es menos importante que el logro educativo? La crítica no es al porcentaje sino al proceso de construcción. Una estadística se legítima cuando nace de una deliberación teóricoconceptual, pero sobre todo participativa, donde los interesados o afectados son los que deben tener voz. El IPM no es una iniciativa nueva, desde el año 2000 hay decenas de propuestas de indicadores multidimensionales. Alrededor del mundo ya no se discute su relevancia, sino cómo consensuar sus componentes. 


\section{Metodología}

La metodología de la investigación es el conjunto de técnicas y métodos que se aplican durante un trabajo de investigación, para poder llegar al resultado de esta. En este sentido, la metodología funciona como parte conceptual que sigue los procedimientos que serán implementados en la investigación. Por lo tanto, en esta sección se presenta el tipo de investigación, los métodos y técnicas que serán empleados, luego se expone de manera específica como realizar el cálculo y toma de la muestra, el proceso de elaboración del instrumento de medición implementado, y por último se detalla el procedimiento para la construcción del índice multidimensional de pobreza para las familias del sector Monte Sinaí.

\subsection{Tipo de investigación}

El tipo de investigación siempre estará en función de las necesidades del autor durante el desarrollo de la investigación, en este caso, para poder conocer las características esenciales del problema fue necesario aplicar investigación descriptiva, con la finalidad de sistematizar la documentación que posteriormente permitiría realizar una descripción estructurada del problema objeto de estudio, que en este caso es el alto porcentaje de familias que viven en condiciones de pobreza y extrema pobreza.

Si bien la investigación descriptiva permite conocer las generalidades del problema, esta no brinda respuesta en cuanto a las causas y efectos del mismo; como solución a esta necesidad, también se aplicó la investigación explicativa, debido a que esta va más allá de una descripción, pues permite obtener una explicación minuciosa sobre los factores que dieron origen al problema, que en este caso es la concentración de la riqueza y el nivel de estudio, que como consecuencia trae la aparición de desigualdades.

Cabe mencionar que como antesala al desarrollo de la investigación se aplicó investigación exploratoria, debido a que se realizó una observación preliminar, con la finalidad de identificar comportamientos o factores que inciden en la problemática; otro motivo es la aplicación de los instrumentos de recolección de información, mismo que fueron aplicados a pobladores de Monte Sinaí.

\subsection{Método de investigación}

En la investigación se implementaron métodos empíricos y teóricos. Entre los métodos teóricos están

Análisis - síntesis, compuesto de dos partes, por un lado está, el análisis que es la separación de un todo en sus partes constitutivas con la finalidad de estudiar cada una de ellas, por separado y a su vez las relaciones que las unen, mientras que por otro lado aparece la síntesis que es la unión de las partes para analizar, dentro de un todo, su naturaleza y comportamiento con el propósito de identificar las características del fenómeno observado.

Se aplica esta metodología tomando en consideración que se analizará la situación socioeconómica de familias del sector de Monte Sinaí, las cuales son referentes de la muestra, adicionalmente se analizarán las diversas dimensiones e indicadores que podrían conformar el índice multidimensional de pobreza que se plantea en este estudio. Tomando de referencias varias fuentes nacionales e internacionales se realizó una síntesis de dichas dimensiones con sus respectivos indicadores, seleccionando así las que conformarían el índice propuesto en esta investigación.

El método Histórico - Lógico, se compone de dos parte, la primera que es la parte histórica que está relacionada con el estudio de la trayectoria real de los fenómenos y acontecimientos, en este trabajo se resumen las diversas aportaciones que han tenido varios autores en torno a la conceptualización de la pobreza. La segunda parte es la lógica que denota el sentido de como las preposiciones que integran una investigación mantienen una interrelación, ser mutuamente excluyentes, por ende deben ser lógicamente consistente; es por ello por lo que la investigación hace referencia a los diversos aportes de la conceptualización de la pobreza por década. 
Planteando un análisis evolutivo del concepto de pobreza, donde la historia se desarrolla a través de una secuencia lógica.

El método deductivo- inductivo, facilita la obtención de conclusiones generales a través de premisas particulares, en el estudio se plantea un análisis a las familias del sector de Monte Sinaí, tomando de referencia a 100 familias de las diversas cooperativas del sector, mismas que van a ser una guía para la aplicación y cálculo del índice multidimensional de la pobreza de un método de medición que proporcionará un resultado general, con lo cual se cumple la parte deductiva; en lo que respecta a la parte inductiva, se debe a la adaptación de este método de medición lo que permitirá generar nuevos enfoques y teorías.

La observación: es un proceso que tiene como función primordial recoger información de manera inmediata sobre lo que se está tratando. Es decir, como este método solo se lo puede aplicar en el momento de la elaboración del trabajo ya sea de campo como en la selección de ciertas variables que solo se las entiende al ser observadas.

Cuando se realizó el trabajo de campo se hicieron varias modificaciones en el instrumento de medición al momento de asistir al lugar que se lo estaba aplicando. La observación muestra la realidad, por ende, ayuda a complementar a los métodos teóricos utilizados en este estudio. Este método se lo utilizó también de manera primordial en la selección de las dimensiones que se utilizan para la creación del índice.

\subsection{Población y muestra}

La población del Monte Sinaí es de 270,000 personas, se dice que como es superior a 100.000 personas se puede considerar como una población infinita por lo que se utiliza la expresión de cálculo para poblaciones infinitas y varianza desconocida, tomando un margen de error permisible de $9.8 \%$ y un nivel de confianza de $95 \%$, niveles de p y q de 0.5 respectivamente, se obtiene una muestra de 100 personas; cabe mencionar que la ejecución del trabajo de campo tuvo lugar en las cooperativas Valle Hermoso, Cerro Porteño, y Los Almendros, que son tres de las 54 que existen en Monte Sinaí con mayor concentración de población.

\subsection{Instrumento de medición}

Como instrumento de medición se decide emplear la encuesta, la cual incorporará preguntas relacionadas a las dimensiones que incorpora el IPM, cabe mencionar que se tomó como referencias instrumentos empleados en estudios relaizados por el INEC, entre los cuales resaltan la Encuesta de condiciones de vida y Encuesta nacional de trabajo infantil. Se extraen de esta encuesta 116 indicadores, el cuestionario fue socializado de manera física a los habitantes de Monte Sinaí.

Para corroborar la confiabilidad, se seleccionó el método estadístico Alfa de Cronbach, donde fue necesario emplear el programa estadístico SPSS, obteniendo un coeficiente $\alpha$ de 0.66 , lo que supone que la encuesta es fiable. En cuanto a la validez del instrumento de medición se aplica el Análisis Factorial Exploratorio, este método determina previamente la medida KMO (Káiser Meyer Olkin), que en este caso resultó ser mayor a 0.5, lo que indica que la relación entre las variables es significativa.

Para confirmar el resultado de KMO, se aplicó la prueba de esfericidad de Bartlett, que contrasta la hipótesis nula de que la matriz de correlaciones de las variables es la identidad, en cuyo caso dichas variables no estarían relacionadas. El resultado reflejó en todos los casos un nivel de significación igual a cero, por lo que se rechaza la hipótesis nula y se confirma que si tiene sentido lógico la estructura dimensional y el análisis factorial efectuado. 


\subsection{Planteamiento del índice de pobreza multidimensional}

Para plantear un índice de pobreza multidimensional se establece en la investigación utilizar siete dimensiones; las cuales van enmarcadas a conocer necesidades, perspectivas, y lo que las familias no poseen, para considerar a una persona como pobre. Considerando las propuestas internacionales y el índice desarrollado en el Ecuador, se realiza una propuesta en esta investigación con siete dimensiones y ciento dieciséis indicadores. Cabe mencionar que estas dimensiones no solo buscan ampliar el concepto en el cual solo se es pobre por ingreso, sino también medir que tipo de beneficios perciben las familias radicadas en Monte Sinaí.

Con base en las siete dimensiones planteadas se elabora un banco de preguntas que las abarcan en su totalidad. Cada pregunta que se efectúa al encuestado se vuelve un indicador, y es así como con cada pregunta se obtiene una respuesta inmediata llegando a obtener una base de datos con 116 indicadores.

\subsection{Procedimiento para el cálculo y el análisis de los resultados del índice de pobreza multidimensional}

El primer paso para elaborar el índice multidimensional es construir una matriz de información primaria, que en el plano metodológico la investigación comienza con la definición de sus objetivos y selección de (i) unidad de análisis (persona de familia encuestada) e indicadores de pobreza multidimensional (j), de tal forma que para cada indicador ( $\mathrm{j}$ ) y cada unidad (i), exista $(\mathrm{x}$ ) valores. Definida esta forma se puede pasar al ordenamiento de los datos, para lo cual se utilizará la forma matricial que es la más operativa, y así se obtiene la matriz de información que se presenta en el Cuadro 2. Los números xij representa los elementos de la matriz establecida, los cuales representan el valor del indicador (j) en la unidad de análisis (i), dicha matriz muestra para cada ij el valor correspondiente, es decir solo debe existir un solo valor xij para cada combinación indicador (j) y personas de familia encuestadas (i).La organización de la información se realizó con la ayuda del tabulador electrónico Excel, para lo cual se creó un libro llamado EPOMU.

Cuadro 2

Matriz de información primaria para datos de encuesta.

\begin{tabular}{|c|c|c|c|c|}
\hline \multirow{2}{*}{$\begin{array}{l}\text { Personas de familias } \\
\text { encuestadas }(=100)\end{array}$} & \multicolumn{4}{|c|}{ Dimensiones de pobreza Multidimensional } \\
\hline & \multicolumn{4}{|c|}{ Indicadores de Pobreza Multidimensional $(\mathrm{j}=120)$} \\
\hline 1 & $\mathrm{X} 11$ & $\mathrm{X} 12$ & ........... & $\mathrm{x} 1 \mathrm{j}$ \\
\hline 2 & $\mathrm{X} 21$ & $\mathrm{X} 22$ & ........... & $\mathrm{x} 2 \mathrm{j}$ \\
\hline .......... & ........... & .......... & ........... & .......... \\
\hline 100 & $\mathrm{Xi} 1$ & $\mathrm{xi} 2$ & ........... & xij \\
\hline
\end{tabular}

Fuente: "Un índice de desarrollo sostenible para ecosistemas

de montaña". Autora: Msc. Lidia Inés Díaz Gispert

El segundo paso es la estandarización de los indicadores por áreas temáticas, para proceder con este paso se debe calcular la media de cada indicador establecido para cada dimensión, así como también con el cálculo de la desviación típica de igual manera por cada uno de los indicadores a utilizar en el índice. Una vez que se obtuvo estos dos valores se procede con la estandarización de los xij de cada persona encuestada, como se indica a continuación: 
Donde:

$$
C i j=\frac{x i j-\bar{X} x i j}{\sigma x i j} \mid
$$

Cij - valor estandarizado

Xij - valor de persona de familia encuestada con respecto a cada indicador de pobreza multidimensional.

$\bar{X} x i j$ - media aritmética de valor total de indicadores (j).

$\sigma x i j$ - desviación típica de valor total de indicadores (j), con respecto a cada persona de familia encuestada.

Luego de estandarizar los valores dados en cada indicador se procede a establecer ponderaciones con respecto a cada indicador de estudio, (Pj); con los valores de la ponderación establecida para cada indicador, obtenido de los resultados de las respuestas otorgadas por los encuestados, se constituye la media ponderada de todas las dimensiones.

Por último se procede con la construcción del índice de pobreza multidimensional, este remite un valor específico de incidencia del indicador analizado por cada individuo encuestado. Este valor específico permite realizar comparaciones entre los diferentes indicadores y por tanto dividir entre diferentes unidades de análisis.

Cuadro 3

Matriz final de valores para el cálculo del IPM

\begin{tabular}{|c|c|c|c|c|c|}
\hline & \multicolumn{4}{|c|}{ DIMENSIONES } & \\
\hline $\begin{array}{l}\text { Indicadores } \\
\text { Encuestados }\end{array}$ & Indj_1 & $\begin{array}{l}\text { Indj_ } \\
2\end{array}$ & $\ldots \ldots \ldots$ & Indj_j & $\sum$ (Pjcij) / $\sum P j$ \\
\hline 1 & c11 & c12 & ............. & $\mathrm{c} 1 \mathrm{j}$ & \\
\hline 2 & $\mathrm{c} 21$ & c22 & ............ & $c 2 j$ & \\
\hline$\ldots \ldots \ldots$ & $\ldots \ldots \ldots$ & $\ldots \ldots \ldots$ & $\ldots \ldots \ldots$ & ........... & \\
\hline $100 \mathrm{i}$ & $\mathrm{C} 1 \mathrm{i}$ & $\mathrm{C} 2 \mathrm{i}$ & ............ & Cij & \\
\hline Ponderaciones & P1 & P2 & ........... & $\mathbf{P j}$ & \\
\hline Índice Global & & & & & Índice Global $=\left[\Sigma\left(\mathrm{Cij}^{*} \mathrm{Pj}\right) / \Sigma \mathrm{Pj}\right] /[\mathrm{N}]$ \\
\hline
\end{tabular}

Fuente: "Un índice de desarrollo sostenible para ecosistemas de montaña". Autora. Msc. Lidia Inés Díaz Gispert

El índice construido constituye en sí, una nueva variable con valores para cada persona encuestada y una vez realizado el cálculo se procedió a tipificarlos de acuerdo con su nivel de pobreza, que en este caso son tres segmentos que son nivel menor, nivel medio y nivel de extrema pobreza.

La clasificación expuesta se logra a través de la ubicación de las personas encuestadas en los distintos intervalos, los mismos que a su vez se calculan partiendo de la expresión " $c$ " que se traduce en la siguiente ecuación $c=$ 1/3[Máx (IPM)-Mín (IPM)]. Conforme se realice la clasificación de encuestados en sus respectivos intervalos, se dará origen a tres grupos de análisis, los cuales contarán con una expresión específica, de manera que la 
expresión de los encuestados de nivel menor es $[M i ́ n(I P M)+C]=1$ er intervalo, la de nivel medio es $[M i ́ n(I P M)+$ $(2 C)]=2$ do intervalo y los de nivel de extrema pobreza es $[$ Má $x(I P M)]=3$ er intervalo.

Con los intervalos establecidos se procede a agrupar a cada familia encuestada en un segmento específico dando como resultado, la clasificación de las familias del sector de Monte Sinaí por niveles de pobreza. La clasificación se la realiza con el tabulador electrónico Excel, el mismo que dependiendo del peso de cada intervalo, brindará un porcentaje a cada rango con valores totales.

\section{Resultados}

Para empezar con el análisis de los resultados de la encuesta, se considera necesario caracterizar las familias radicadas en Monte Sinaí, que es un sector ubicado al noroeste de la ciudad de Guayaquil, habitado por 270.000 habitantes, de los cuales el $99.97 \%$ vive en condiciones de pobreza, cuentan con servicios básicos limitados, como lo son el alcantarillado y el acceso al agua potable.

Para desarrollar este epígrafe se mencionará las siete dimensiones, donde se expresarán los indicadores de mayor relevancia, que permitan entender el nivel de pobreza en el que viven estas familias, donde la representación numérica ayudará a entender las privaciones de este grupo de habitantes.

\subsection{Dimensión formación de familia}

La dimensión está conformada por once indicadores que reflejan cómo está compuesta la familia, número de integrantes, que nivel de educación prevalece en el hogar, entre otros. De los once indicadores se realizará el análisis en dos grupos, el primer grupo buscará analizar la composición numérica de las familias y el segundo grupo trata puntos relacionados con la educación, profundizando así, los resultados más relevantes de la dimensión.

Los resultados arrojan que más del $50 \%$ de las familias de Monte Sinaí, consta de aproximadamente de 5 a 6 integrantes de los cuales de 2 a 3 son personas que no generan ingresos, menores a 16 años y 2 a 3 personas actas para generar ingresos. En cuanto a educación se evidencia que el $85 \%$ de las familias mantienen miembros con un nivel de educación secundario, el $9 \%$ mantienen una educación primaria y solo el $6 \%$ mantienen una educación de tercer nivel, se puede decir que estudiaron una tecnología. Cabe mencionar que existen familias que presentan dificultades para acceder a la educación básica ya sea por falta de recursos, como de motivación por parte del núcleo familiar.

\subsection{Dimensión salud y nutrición}

Esta dimensión contiene nueve indicadores, entre los cuales constan, su acceso a la salud pública, la tenencia de seguros de vida, la frecuencia con la que se agendan consultas y por último, posibles enfermedades que les signifiquen un impedimento para realizar sus actividades. Esta dimensión se la dividirá en dos partes la primera enfocada a identificar como las familias cubren la necesidad de atención médica, que prácticas ellas mantienen para resolver sus problemas de salud y cuantas comidas al día consumen en promedio; y la segunda parte a detallar que perspectiva ellos tienen de la salud que mantienen los miembros de la familia.

Los datos arrojados en la encuesta indican que existe un $49 \%$ de personas que indicaron que, si sufrieron algún quebranto en la salud; sin embargo, lo preocupante es que el $36 \%$ de las personas que sufrieron un quebranto en su salud acudieron al médico y del $64 \%$ restante, un $33 \%$ no acuden y un $31 \%$ acuden a veces, lo que supone que las personas que sufren alguna dificultad en su salud no reciben o buscan la atención médica necesaria.

El $64 \%$ de habitantes que no acude al médico, opta por tomar otras alternativas donde el $31 \%$ elige automedicarse, el $29 \%$ consultan al farmacéutico, que son personas que en la mayoría de los casos no cuenta 
con los conocimientos necesarios para poder medicar a una persona, ya que no son médicos y el $19 \%$ espera a que la enfermedad ceda.

Entre las razones por las que el $64 \%$ de habitantes no acude al médico, están la falta de tiempo con un $34 \%$ la de mayor relevancia, la falta de dinero con un $25 \%$ y $16 \%$ indica que el servicio al que acceden es malo. En cuanto al servicio médico al que acceden estas familias se tiene que el $71 \%$ de los integrantes de una familia acuden a un dispensario del MSP (Ministerio de Salud Pública), mientras que, 22\% hacen uso del servicio a través de hospitales públicos y $7 \%$ son personas que cuentan con el seguro social y además pueden atenderse en hospitales privados sin ningún costo adicional.

El 54\% de las familias consumen entre 2 a 3 comidas poco nutritivas al día y un $44 \%$ de las familias consumen entre 4 a 5 comidas poco nutritivas al día, según datos del MIES, el sector de Monte Sinaí es uno de los más vulnerables a nivel de nutrición de niños y niñas. Con respecto a la Percepción de la salud en el núcleo familiar, se pudo conocer que $65 \%$ de las familias consideran la salud de los niños como buena, mientras que, $61 \%$ de las familias considera que la salud de los adultos es buena. Esta percepción de cada núcleo familiar depende de la condición que se encuentra en el momento de aplicar la encuesta; sin embargo, se considera que indicar el estado de saludo como "Buena" no es señal de que gocen de una salud óptima.

\subsection{Dimensión de políticas de gobierno}

Esta dimensión está conformada por 6 indicadores los cuales buscan descubrir los resultados a dos programas implementados por el gobierno a las familias pobres, entre los indicadores está la percepción del Bono de Desarrollo Humano y el destino que le dan a dicho recurso, además de la existencia de integrantes de la familia con discapacidad física o mental. En el sector de Monte Sinaí, un 35\% de las familias reciben el BDH, de las cuales el $31 \%$ destina este recurso a la compra de alimentos. En cuanto a temas de discapacidad física o mental, el $31 \%$ de las familias cuenta con un integrante con algún tipo de discapacidad física y de esta solo el $10 \%$ es beneficiaria del bono por discapacidad.

\subsection{Dimensión de hábitat y ambiente sano}

Esta dimensión contiene 20 indicadores los cuales contemplan el entorno de las familias del sector. Cómo estas familias viven, cuáles son los riesgos a los que se exponen a diario y sobre todo qué perspectiva mantienen acerca de la calidad de sus hogares. Para realizar el análisis de esta dimensión se la segmentará en dos partes, la primera unificará el análisis del estado de la vivienda, la segunda incluirá los indicadores que reflejan las condiciones de hábitat de las familias en el sector.

En lo que respecta al análisis del estado de las viviendas el 93\% vive en casas, donde el material que predomina en un $67 \%$ es el cemento y el $30 \%$ es la caña o tabla. En el piso el material que predomina es el cemento con un $45 \%$ mientras que un $24 \%$ es de tablas. Adicional cuando se consultó sobre el estado del techo de la vivienda un $51 \%$ consideran que el estado es regular y un $41 \%$ es bueno.

En la búsqueda de las opiniones de cada familia acerca de su vivienda se consultó que calidad consideran que tiene la misma, el $61 \%$ indicó, que la considerada buena, un $24 \%$ que la considera regular. Otra de las consultas realizadas en el cuestionario es sobre el espacio de sus viviendas en relación a las personas que habitan en ellas, teniendo así un $63 \%$ que consideran el espacio donde habitan es suficiente para la cantidad de miembros de la familia.

Con respecto al hábitat de las familias del sector, fue posible evidenciar que las vías de acceso a las viviendas en un $89 \%$ son una calle de tierra, y pocas las calles principales que se encuentran pavimentadas. Por otra parte, un 
$63 \%$ las familias indican que no hay asentamientos cercanos a su hogar, sin embargo, el $58 \%$ de las familias consultadas consideran que el sector se encuentra sobrepoblado.

Al consultarles sobre el acceso al agua potable en el sector un $59 \%$ de familias si tienen acceso y un $41 \%$ no tiene acceso al servicio, esto se debe a que el servicio de alcantarillado aún no está concluido en este sector, como dato adicional se utiliza pozo séptico para las aguas servidas. El análisis arrojó que el $71 \%$ de las familias hierven el agua antes de consumirla. De igual forma se consultó si en algún momento se ha evidenciado cualquier tipo de plagas en los hogares, donde se obtuvo como respuesta que un $79 \%$ si ha evidenciado la existencia de estas, de los cuales el $47 \%$ indican que existen ratones y un $24 \%$ que existen cucarachas.

EI MSP, continuamente ofrece programas de prevención de plagas, sobre todo en las zonas más vulnerables sobre ello, también se consultó a las familias del sector, las mismas que indicaron que un $56 \%$ desconocen de campañas de este tipo, no se habían acercado a ofrecer alguna información de cómo prevenir las plagas e incluso indican que las campañas de fumigación son muy escazas. Por otro lado, se consultó si existía algún tipo de contaminante cerca de los hogares dando como resultado que un $34 \%$ de las familias indicaron que existe un botadero de basura a pesar de que el servicio de recolección de basura municipal lo califican como bueno un $68 \%$ de familias, las personas del sector no respetan los horarios y originan estos botaderos improvisados de basura. Al ser consultados sobre el tipo de riesgo al que las familias del sector que son expuestas de manera permanente, un $51 \%$ consideran que el mayor riesgo es la delincuencia.

\subsection{Dimensión trabajo y seguridad social}

En esta dimensión se incorporaron 20 indicadores que reflejan el estado laboral del jefe de hogar, que cantidad de personas son desocupadas, amas de casa y estudiantes. También muestra si el jefe de hogar cuenta con la afiliación al seguro social y por ende accesibilidad a los servicios que el mismo brinda. Para realizar el análisis de esta dimensión se agrupa los indicadores en dos segmentos el trabajo donde se analizan aspectos tales como tipo de empleo, características del empleo, cantidad de personas empleadas, desocupadas, amas de casa del hogar, entre otros indicadores. El segundo segmento será de Seguridad Social, donde se evalúan aspectos tales como una afiliación con el seguro social y qué tipo de información mantienen acerca del mismo.

En el segmento de trabajo los resultados reflejan que un 32\% de los núcleos encuestados no mantienen personas desocupadas, mientras que un $26 \%$ de las familias cuentan con solo 1 persona desocupada, si se analiza de manera global se puede decir que, en los núcleos familiares del sector de Monte Sinaí el promedio de personas desocupadas es mínimo, este indicador se podría interpretar como bueno. El $44 \%$ de las familias poseen dos miembros que son estudiantes, el $16 \%$ indicaron que tenían tres estudiantes en el núcleo familiar. En el $77 \%$ de los hogares solo hay una ama de casa.

Solo el jefe de hogar es el que aporta económicamente para que la familia pueda suplir sus necesidades en general, es decir que no poseen un ingreso adicional para compensar estudios, vestimenta, salud. El $61 \%$ de jefes de hogar poseen un empleo formal, solo el 32\% posee un empleo informal, ahora bien, del $32 \%$ de los trabajadores informales solo el $15 \%$ consideran que la ganancia percibida les cubre un día de alimentación y el $17 \%$ restante indica que no se otorgan las facilidades para poder realizar su actividad. En el segmento de seguridad social, los resultados indican que el $66 \%$ es afiliado al IESS, es decir, que la mayor parte de los habitantes reciben atención a través del IESS, un 54\% de las personas tienen aportando menos de 1 año, lo que supone que aún no poseen una estabilidad laboral, abriendo la posibilidad de perder su empleo.

\subsection{Dimensión ingreso, ahorro e inclusión financiera}

La siguiente dimensión hará referencia al ingreso y egreso de las familias del sector de Monte Sinaí, mostrará si las familias reciben alguna ayuda económica adicional a la ganancia percibida por el jefe de hogar, se mostrará si poseen ahorros o si han adquirido algún tipo de crédito, para todo esto se establecieron 24 indicadores. Para realizar el análisis de esta dimensión los indicadores serán divididos en dos grupos, el primer grupo buscará 
reflejar los ingresos y egresos de las familias. El segundo grupo mostrará cómo es la situación financiera dentro de la familia.

Los resultados de los indicadores de ingresos indican que el valor mínimo de ingreso mensual en una familia es de $\$ 60.00$ mientras que el valor máximo es de $\$ 750.00$, con lo cual el promedio mensual de ingresos en estas familias es de $\$ 398.56$, en este valor se incluye no solo los sueldos percibidos por los jefes de hogar, incluye los ingresos extras como subsidios otorgados por el gobierno como el BDH, incluye las pensiones de jubilación que cuentan las familias como aporte de las personas de tercera edad jubiladas.

Los resultados de los indicadores de egresos muestran que en promedio las familias destinan $\$ 216,55$ para la compra de bienes; esto se refiere a los productos que consumen en su alimentación, salud, educación, vestimenta, y demás bienes que pueden llegar a necesitar. El valor promedio destinado a pago de servicios básicos es de $\$ 34.92$, esto considerando luz, agua que reciben en un tanquero pasando 2 días y en algunas familias teléfono, generalmente cuentan con este servicio las familias que tienen un pequeño negocio en su domicilio.

En lo que refiere al grupo de Inclusión financiera, se consultó si habían recibido algún tipo de crédito en los últimos 12 meses y cuál había sido la fuente de este, los resultados muestran que el $45 \%$ no habían recibido este tipo de crédito, el $29 \%$ si realizaron un crédito a terceras personas, no en una institución financiera y el $26 \%$ indicó haber obtenido el crédito de un banco privado o estatal.

De este $55 \%$ de personas que realizaron un crédito, el $29 \%$ lo destino para pago de alimentación, estudio o arreglo de vivienda, el 13\% realizó el pago de deudas con el dinero prestado y el 12\% realizó la inversión en un emprendimiento o en alguna emergencia médica. Adicional, se consultó si contaban con cuenta de ahorros, con lo cual, fue posible conocer que el $48 \%$ de personas mantienen este producto financiero. Al consultarles si mantenían algún tipo de ahorro en las mismas, el $60 \%$ expresó que no mantenían ahorros y solo un 33\% indicaron que tenían una pequeña cantidad de dinero guardada para casos emergencia.

\subsection{Dimensión de percepción de calidad de vida}

Para analizar esta dimensión se segmentará a los indicadores en tres grupos, el primer grupo buscará medir la perspectiva de la calidad de vida que tienen las familias del sector, en el segundo grupo se presentará la perspectiva de pobreza y de bienestar que tienen las mismas familias; y por último se analizará qué dimensión utilizada en esta encuesta considerada por las familias es más importantes para mejorar la calidad de vida, con vista a usar estas opiniones como ponderadores en el índice final.

En cuanto a la perspectiva de la calidad de vida, los resultados arrojan que el $49 \%$ si dedica tiempo para realizar alguna actividad de recreación. Sobre su satisfacción con su trabajo el $63 \%$ se encuentra conforme y el 36\% indicaron que no. Entre los motivos de dicha insatisfacción están, los salarios con $13 \%$ y la escasez de oportunidades de desarrollo laboral con $9 \%$.

Al abordar el tema de la discriminación se obtuvo como resultado que el $43 \%$ de los habitantes sufrieron discriminación de algún tipo, siendo las más comunes la discriminación por nivel de educación en un $20 \%$ de los casos y $9 \%$ por su etnia. Con respecto al transporte público un $49 \%$ de las personas indicaron que en general sufren dificultades con el transporte, las líneas de buses que ingresan a estos sectores solo transitan hasta un tiempo determinado y que no existe seguridad en las mismas.

En lo referente a la perspectiva de la pobreza, los resultados indican que el $61 \%$ de las personas encuestadas se consideran pobre y el $20 \%$ se consideran medianamente pobre. Sobre la percepción que tienen sobre el bienestar social, el $62 \%$ de los habitantes consideran que en su condición de vida actual mantienen poco bienestar, el $34 \%$ indica que mantiene un bienestar medio, y solo el $4 \%$ de las familias sentían mucho bienestar. 


\subsection{Determinación del IPM según el procedimiento propuesto}

Como primer paso se obtiene la matriz primaria en la cual se ingresan los resultados obtenidos en las encuestas según la división de dimensiones e indicadores con la codificación asignada para el análisis de los datos en el aplicativo estadístico SPSS, también se muestran el número de familias ingresadas que para la muestra son 100 como ya se indicó. El segundo paso es donde se realiza la estandarización de los valores de la matriz primaria se utilizó la forma de estandarización general, dando como resultados valores dentro de un mismo intervalo; teniendo así la matriz lista para realizar el cálculo multidimensional. Para el tercer paso tenemos la transformación de la matriz primaria aplicando las ponderaciones que le otorgaron las familias del Sector Monte Sinaí a cada dimensión, esto con base en el resultado de la última pregunta del cuestionario que recaba la opinión de estas familias sobre las dimensiones que tienen mayor peso en su situación actual.

Luego de plantear las matrices utilizadas en el estudio se establecen intervalos para designar qué porcentaje de la población del sector de Monte Sinaí se encuentran es extrema pobreza, pobreza media y baja pobreza, el valor mínimo -2,62 y máximo 3,27 de los índices de las 100 familias encuestadas en el sector; luego se procede a calcular la variable "c", Una vez calculada la variable c, se procede con el cálculo de los valores que formaran parte de la asignación para delimitar cada intervalo, para luego poder segmentar en los tres grupos a las familias.

\subsection{Análisis de los resultados del IPM para las familias de Monte Sinaí}

En la comprobación con pruebas estadísticas es posible apreciar que, la media y la mediana valor que divide a la población en este caso a las familias del sector de Monte Sinaí, en dos grupos iguales, (media: 0,000; mediana: $0,08)$, toman valores muy próximos indicando un cierto grado de simetría. Sin embargo, no se puede decir que la distribución del indicador sea la típica distribución Normal, simétrica con respecto a la media y en la que la media, mediana y moda coinciden. Con todo, el contraste de normalidad de la prueba de Kolmogorov Smirnov, conduce a aceptar la hipótesis nula de Distribución Normal: el estadístico Z de Kolmogorov Smirnov toma el valor de 0,915, mismo que tiene asociado un nivel de significación igual a 0,372; que significa que para cualquier nivel de significación que se tome se acepta la hipótesis nula de que la distribución del indicador es normal.

Para realizar el análisis del IPM, se definieron intervalos para cada categoría en el caso de la categoría de menor pobreza el intervalo es de $[-2,62 ;-0,65]$, para la categoría de pobreza media el intervalo es de $]-0,65 ; 1,32]$ y para la categoría de nivel de extrema pobreza el intervalo es] 1,32; 3,29].

La categoría de menor pobreza, se encuentra conformada por 30 familias, de las cuales 18 familias están sobre la línea de tendencia la cual indica que si llegan a tener alguna necesidad sin satisfacer o un indicador llega a desmejorar, son propensas a cambiar de intervalo e incrementar su IPM. El índice promedio en las familias de esta categoría está entre -1,5; -1,00. Entre las características generales de esta categoría se tiene que:

- En las familias predomina un promedio de 4 miembros, en las cuales el $60 \%$ se presentan sin hijos o solo con un hijo menor de 16 años. Existen $40 \%$ familias, que están formadas por madre y padre (nuclear). El tipo de escolaridad que predomina es el bachillerato con un $90 \%$ que pertenecen a 26 familias de las 30 integradas en este intervalo. El $63 \%$ de familias que necesitan atención medica acuden a los dispensarios del MSP.

- Por otro lado, el $67 \%$ de familias consume de dos a tres comidas poco nutritivas, el $30 \%$ indica que consume de cuatro a cinco comidas al día no tan nutritivas. En este grupo el $50 \%$ cuenta con la ayuda del BDH. De ello el $43 \%$ lo invierten en alimentación. En las viviendas el $60 \%$ de material que predomina es el cemento, un $50 \%$ de personas cataloga a la calidad de la vivienda como buena.

- El 83\% de las familias tiene como vía de acceso principal una calle de tierra. El 63\% de familias reciben agua potabilizada. El $57 \%$ de jefes de familias mantienen una actividad laboral informal, con un ingreso promedio mensual de $\$ 351,00$; sin embargo, al analizar este valor considerando la cantidad de miembros de las 
familias que como promedio es de 4 , el ingreso percápita de estas es de $\$ 87.75$ promedio mensual. El 50\% de familias son afiliadas al seguro social, donde el $60 \%$ mantiene una afiliación reciente hasta un año. Cuando se consultó sobre su percepción de considerarse pobres el $87 \%$ de las familias indicaron que si se consideran pobres y el $63 \%$ indicó que perciben poco bienestar.

La categoría de pobreza media, la integran 53 familias, en este caso se muestra la línea de tendencia a la baja es decir que existen una mayor cantidad de casos de familia que podrían estar pasando de una pobreza media a una menor pobreza, esto depende mucho de la modificación de los indicadores, de que puedan llegar a cubrir alguna necesidad que actualmente la mantienen insatisfecha. Las características generales de estas familias son:

- El 30\% de familias están integradas de 5 miembros. De los cuales, el 42\% mantienen dos niños de hasta 16 años. El nivel de escolaridad que predomina es el bachillerato con un $85 \%$; sin embargo, existe un $19 \%$ de familias que mantienen un integrante analfabeto. El 74\% indicó que acuden al dispensario del MSP cuando presentan algún quebranto en la salud; sin embargo, el 34\% indicó que el servicio al cual acuden es malo. El consumo de comida en este grupo de familias, indica que un $51 \%$ consumen de dos a tres comidas poco nutritivas.

- Al menos del 21\% de familias que reciben el BDH, el 23\% lo destina para la alimentación. El 92\% de familias tienen como vía de acceso a sus hogares una calle de tierra. El material que predomina en las viviendas es el cemento con un $62 \%$, donde el $66 \%$ de las familias catalogan la calidad de la vivienda como buena. El $38 \%$ de familias indicaron que si existen asentamientos cerca a su hogar. Incluso el $57 \%$ indica que si consideran el sector donde viven sobrepoblado.

- El $57 \%$ de familias recibe agua potable. Respecto a la presencia de plagas el $74 \%$ indicó que en su hogar había presenciado algún tipo de plaga, donde el $47 \%$ indicó que la plaga frecuente eran las ratas. El $70 \%$ de jefes de hogar trabaja en relación de dependencia, solo el $23 \%$ de familias trabaja de forma informal y el ingreso estimado mensual es de $\$ 411,00 ;$ sin embargo, al analizar este valor considerando la cantidad de miembros de las familias que como promedio es de 5, el ingreso percápita de estas es de $\$ 82.2$ promedio mensual. La afiliación al seguro social la mantiene el $77 \%$ de jefes de hogar de los cuales un $49 \%$ mantienen una afiliación de hasta un año. El $47 \%$ de las familias se consideran pobres y el $57 \%$ mantienen poco bienestar.

Por último está la categoría de pobreza extrema que la integran 17 familias, donde es posible apreciar como la línea de tendencia se encuentra a la baja es decir la tendencia a reducir la pobreza la mantiene todas las familias, de los 17 casos 9 se encuentran por encima de la línea de tendencia. Existe un caso con un índice de 3,27 que sería la familia con mayor pobreza extrema. Los rasgos generales de estas familias son:

- En este grupo el tipo de familias que predomina son las de abuelos con un $35 \%$ y las monoparentales también con un $35 \%$. Existe un $47 \%$ de familias que están conformadas por 7 miembros; de los cuales el 47\% mantienen 2 miembros de hasta 16 años. El nivel de escolaridad que predomina en las familias del sector es bachillerato con un $76 \%$.

- El 76\% de las familias indican que al sufrir algún quebranto en su salud acuden al MSP, y el $41 \%$ mencionan que no acuden a centros de salud por la falta de tiempo. El 35\% de familias indican recibir el BDH, el mismo que es utilizado en un $35 \%$ a la compra de alimentos. La calidad de la vivienda es bueno en un $65 \%$ de las familias, mientras que en un $24 \%$ el estado es regular.

- El 88\% de las vías de acceso a la vivienda es de tierra, calles sin pavimentar. Cuando se consultó sobre la presencia de plagas, el 100\% mencionó que existían plagas en su hogar, donde el $65 \%$ indicaron que había ratas, el $24 \%$ cucaracha y el $12 \%$ mosquitos o moscas. En lo laboral el $18 \%$ trabaja de manera informal, el $53 \%$ dependiente. 
- El promedio de ingreso mensuales es de \$447,00 para la familia; sin embargo, al analizar este valor considerando la cantidad de miembros de las familias que como promedio es de 7 , el ingreso percápita de estas es de $\$ 63.85$ promedio mensual. Existe un $59 \%$ de jefes de hogar que se encuentran afiliados al seguro, sin embargo, el $71 \%$ indicó que no conocen los servicios que ofrece el seguro social e incluso el $82 \%$ indicó que no utilizan ninguno de los servicios del seguro. En las respuestas de percepción al consultar sobre si se consideraban pobres o no, el $53 \%$ indicó que si se consideran, el $24 \%$ que no se consideran pobres. La pregunta sobre el grado de bienestar el $76 \%$ indicó que posee poco bienestar.

\section{Conclusiones}

Se logra sistematizar los diversos conceptos de pobreza de forma cronológica exponiendo así los aportes de representantes de las escuelas de pensamiento económicas y de organizaciones mundiales, mencionando también los diversos métodos de medición. Se concluye que la pobreza es un concepto multidimensional que incorpora varios enfoques para así establecer ciertas características por las cuales se considera como pobre a una persona no sólo por la insatisfacción de sus necesidades básicas; sino por su calidad de vida.

Se logró establecer un procedimiento metodológico para la construcción del IPM, implementando métodos estadísticos que permitieron su medición y aplicación a las familias del sector de Monte Sinaí. El procedimiento para el cálculo del IPM que se propone es coherente e integra dos procedimientos utilizados ya en otros estudios.

Se cumple el objetivo general planteado en la investigación ya que fue posible determinar un índice de pobreza multidimensional con 7 dimensiones y 116 indicadores, lo que permitió evidenciar las causas socioeconómicas que mantienen a las personas en una situación de pobreza. Como resultado de este índice se logra clasificar las familias del Sector Monte Sinaí en tres intervalos de la siguiente manera: 30\% Familias fueron clasificadas en menor pobreza, 58 \% se clasifican en pobreza media y $17 \%$ en pobreza extrema. Para validar el índice se aplicó la prueba estadística de Kolmogorov Smirnov obteniendo como resultado que en la distribución del IPM existen $58 \%$ familias que presentan valores centrales en torno a la media. El estadístico Z de Kolmogorov Smirnov toma el valor de 0,915, mismo que tiene asociado un nivel de significación igual a 0,372, lo que supone que significa para cualquier nivel de significación que se tome se acepta la hipótesis nula de que la distribución del indicador es normal.

Al comprobar la hipótesis de investigación se pudo constatar que las características socioeconómicas de las familias del sector de Monte Sinaí, pertenecientes al grupo de "menor pobreza" son que el $90 \%$ de las familias presentan un nivel de escolaridad secundario, el $50 \%$ de la familias cuentan con la ayuda del BDH, el $57 \%$ de jefes de familias mantienen una actividad laboral informal, con un ingreso promedio mensual de $\$ 351,00$, el $50 \%$ de familias son afiliadas al seguro social, donde el $60 \%$ mantiene una afiliación reciente de hasta un año. Las familias que pertenecen al grupo de "pobreza media" presentan las siguientes; el nivel de escolaridad que predomina en este grupo es el bachillerato con un $85 \%$; sin embargo, existe un $19 \%$ de familias que mantienen un integrante analfabeto, el $70 \%$ de jefes de hogar trabaja en forma de dependencia, solo el $23 \%$ de este grupo de familias trabaja de forma informal; el ingreso estimado mensual es de $\$ 411,00$, el $38 \%$ de familias indicaron que si existe asentamientos cerca a su hogar. Adicional, estos dos grupos presentan características similares descritas a continuación: algunas familias no superan a 4 integrantes, son familias de tipo nuclear o reconstruida, presentan accesibilidad superior al $50 \%$ de agua potable. Finalmente, las familias que integran el grupo de "pobreza extrema" presentan estas características; el tipo de familias que predomina son las de abuelos con un $35 \%$ y las monoparentales también con un 35\%. El $47 \%$ de familias están conformadas por 7 miembros; el nivel de escolaridad que predomina es bachillerato con un 76\%, En lo laboral el 18\% trabaja de manera informal; el 53\% indican que trabajan de manera dependiente; ahora bien, sin embargo, existen el 35\% de desocupados por núcleo familiar, el promedio de ingresos mensuales es de $\$ 447,00$ para cada familia. De tal forma que la medición 
proporcionada brinda la información necesaria para indicar que tipo de pobreza mantiene cada una de las familias y cuáles son las variables de mayor peso.

El sector que fue objeto del estudio presentó los rasgos visibles para considerar la aplicación de un IPM, los resultados contrastan claramente con la realidad, considerando que el Sector de Monte Sinaí es uno de los más pobres en la ciudad de Guayaquil. Cuando se realizó la comparación con el índice que se calcula en el Ecuador. El cual da como resultado que en Guayaquil existe la cantidad de 389,470.000 personas que viven en condiciones de pobreza, según datos calculados a detalle en la investigación, si a este valor se le calcula el 12,00\% que representa la población de Monte Sinaí se obtiene como resultado que solo 46,736.40 son considerados como pobres y extremadamente pobres; sin embargo, como ya se lo indicó el 99,97\% de los habitantes del sector son considerados como pobres. La diferencia de habitantes es de 223,263.60 que no consta en esas cifras en las fuentes oficiales. Es así como el índice de pobreza por ingresos que ofrece las fuentes oficiales enmascara la realidad del sector.

\section{Referencias bibliográficas}

Añazco, R. C., \& Pérez, F. J. (2016). Medición de la Pobreza Multidimensional en Ecuador. Obtenido de https://www.ecuadorencifras.gob.ec/documentos/webinec/Sitios/Pobreza_Multidimensional/assets/ipm-metodologia-oficial.pdf

Cruz, B. V. (2016). El nuevo Índice de Pobreza Multidimensional: lo bueno y lo malo. Obtenido de https://www.eluniverso.com/opinion/2016/02/16/nota/5407699/nuevo-indice-pobrezamultidimensional-bueno-malo/

Dávila, S. B.; Ortega, F. C. (2015). Pobreza multidimensional en Ecuador: Aplicación del Índice de Pobreza Multidimensional de Alkire y Foster para Ecuador 2008-2014. Obtenido de http://foroeconomiaecuador.com/fee/download/Burgos-y-Cando-2015.pdf

Guerrero Mills, M. (2015). Las principales escuelas del pensamiento económico en relación con la Ley de Pobres. Obtenido de https://www.academia.edu/9371063/Las_principales_escuelas_del_pensamiento_econ\%C3\%B3mico_en_ relaci\%C3\%B3n_con_la_

Sampiere, R. H. (2014). Metodología de la investigación. En R. H. Sampiere, Metodología de la investigación. México: McGRAW-HILL / Interamericana Editores, S.A. DE C.V. 90.

SEN, A. (1976). Poverty: An Ordinal Approach to Measurement. Econometrica. 44(2), 219-231. doi:https://doi.org/10.2307/1912718

Serrano, M. (2017). Reporte de Pobreza y Desigualdad 2017. Obtenido de https://www.ecuadorencifras.gob.ec/documentos/webinec/POBREZA/2017/Junio/Informe\%20pobreza\%20y\%20desigualdad\%20\%20jun\%202017\%2014072017.pdf

Esta obra está bajo una Licencia Creative Commons Attribución-NoCommercial 4.0 International

(cc) BY-NC 